\title{
Caracterización clínico-epidemiológica de las enterocolitis necrosantes neonatales de siete hospitales públicos
}

\section{Clinical-epidemiological characterization of neonatal necrotizing enterocolitis of seven public hospitals}

\author{
Alejandra Sandoval C. 1,9, Fernanda Cofré S. ${ }^{2,9}$, Mariluz Hernández E.4,9, Giannina Izquierdo C. 5,9, Yenis Labraña C. 6,9, \\ Alejandra Reyes J.7,9, Héctor Pérez A. ${ }^{1}$, Scarleth Brethauer M. ${ }^{2}$, Miguel A. Pantoja H. ${ }^{3}$, Cristina Lindemann T. ${ }^{4}$, \\ Gustavo Orellana S. ${ }^{5}$, Carolina Méndez B. ${ }^{6}$, Adela Figueroa B. ${ }^{7}$, Jaime Cerda L. ${ }^{8}$ y Luis Delpiano M..$^{3,9}$
}

\author{
${ }^{1}$ Complejo Asistencial Dr. Sótero del Río. Santiago, Chile. \\ ${ }^{2}$ Hospital San José. Santiago, Chile. \\ ${ }^{3}$ Hospital Clínico San Borja Arriarán. Santiago, Chile. \\ ${ }^{4}$ Hospital La Florida Dra. Eloísa Díaz. Santiago, Chile. \\ ${ }^{5}$ Complejo Asistencial Barros Luco Trudeau. Santiago, Chile. \\ ${ }^{6}$ Hospital San Juan de Dios. Santiago, Chile. \\ ${ }^{7}$ Hospital Félix Bulnes Cerda. Santiago, Chile. \\ ${ }^{8}$ Departamento de Salud Pública, Pontificia Universidad Católica de Chile. Santiago, Chile. \\ Infectólogos Pediatras, Comité Consultivo de Infecciones Neonatales Sociedad Chilena de Infectología.
}

Los siguientes autores tienen actualmente otra filiación:

Alejandra Sandoval C. Hospital Carlos Van Buren, Valparaíso, Chile.

Mariluz Hernández E. Hospital Santiago Oriente Luis Tisné Brousse, Santiago, Chile.

Los autores no presentan conflictos de interés.

No hubo financiamiento externo.

Recibido: 15 de noviembre de 2019 (segunda versión: 21 de septiembre de 2020) / Aceptado: 21 de septiembre de 2020

\section{Resumen}

Introducción: Enterocolitis necrosante $(\mathrm{ECN})$ representa una elevada mortalidad y morbilidad post-quirúrgica, gastrointestinal y del neuro-desarrollo. Existe limitada información en Chile. Objetivo: Describir el comportamiento clínico/epidemiológico de recién nacidos que cursaron con ECN. Pacientes y Métodos: Estudio multicéntrico descriptivo de pacientes con ECN de siete hospitales de Santiago, Chile, durante el 2016. Se realizó estadística descriptiva y análisis univariable/multivariable (software SPSS v22). Resultados: Se recolectaron 75 casos. Mediana de edad al diagnóstico fue 11 días, el promedio de edad gestacional 29 semanas y peso de nacimiento $1.285 \mathrm{~g}$. La incidencia fue 2,6 por 1.000 /recién nacidos vivos y letalidad de $18,6 \%$, mayor en $\leq 750 \mathrm{~g}, \leq 25$ semanas y ECN quirúrgica. Hubo aislamiento microbiológico en $45,3 \%$ y se utilizaron 19 distintos esquemas antimicrobianos empíricos para el tratamiento de ECN. El análisis multivariable mostró tendencia a que la ECN fuese quirúrgica

\begin{abstract}
Background: Necrotizing enterocolitis (NEC) presents high mortality and postoperative, gastrointestinal and neurodevelopmental morbidity. There is limited information about NEC in Chile. Aim: To describe the clinical/epidemiological behavior of newborns who underwent NEC. Methods: Multicenter descriptive study of patients with NEC from seven hospitals of Santiago, Chile, during 2016. Descriptive statistics and univariate/multivariate analysis were performed (SPSS v22 software). Results: 75 cases were collected. Median days of life at diagnosis was 11, gestational age was 29 weeks, birth weight $1,114 \mathrm{~g}$. The incidence was 2.6 per 1,000 live newborns and mortality was $18.6 \%$, higher in $\leq 750 \mathrm{~g}, \leq 25$ weeks and surgical NEC. There was $45.3 \%$ microbiological isolation and 19 different empirical antibiotic schemes were used for the treatment of NEC. The multivariate analysis showed a higher risk of surgery in umbilical arterial catheter users, $\mathrm{CRP}>10 \mathrm{mg} / \mathrm{L}$ and positive microbiological
\end{abstract}


en usuarios de catéter umbilical arterial, $\mathrm{PCR}>10 \mathrm{mg} / \mathrm{L}$ y aislamiento microbiológico, y hubo tendencia a fallecer en usuarios de catéter umbilical arterial. Discusión: Es el primer estudio multicéntrico que recopila información de datos locales. La incidencia fue similar a la descrita en la literatura médica, en cambio la letalidad fue algo menor. No existen consensos del tratamiento antimicrobiano a utilizar. Con estos resultados esperamos avanzar en mejorar el diagnóstico y unificar tratamientos antimicrobianos, para reducir cifras de morbimortalidad.

Palabras clave: enterocolitis necrosante, microbiota intestinal, lactancia materna, aislamiento mcrobiológico, antibioterapia empírica.

\section{Introducción}

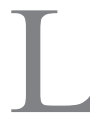
a enterocolitis necrosante (ECN) es una enfermedad grave e impredecible que afecta a los recién nacidos prematuros (RNPT) y es la emergencia gastrointestinal más común observada en unidades de cuidados intensivos neonatales (UCIN). A pesar de décadas de investigación, la patogenia y fisiopatología de esta enfermedad multifactorial sigue sin estar del todo clara, el diagnóstico puede ser difícil y el tratamiento continúa siendo un reto ${ }^{1-10}$. En los últimos años, con el advenimiento de los estudios de secuenciación, la investigación se ha focalizado en la identificación del desequilibrio de la microflora intestinal y disbiosis, ya que, la ECN parece ser el resultado de una respuesta hiperinflamatoria a la microbiota intestinal ${ }^{11,12}$.

Se estima que 2 a $5 \%$ de todas las admisiones en UCIN en todo el mundo se deben a ECN. El 85 a 90\% de los casos ocurre en los RNPT $<1.500 \mathrm{~g}$ y/o $<32$ semanas $^{3,13}$. La incidencia es de 1 a 3 por 1.000 recién nacidos vivos $(\mathrm{RNV})^{(14-17)}$, siendo inversamente proporcional a la edad gestacional $(\mathrm{EG})^{3,18}$.

La ECN tiene una letalidad de 15 a $30 \%$ y en ECN complicadas que requieren resolución quirúrgica puede elevarse hasta 40 a $50 \%$, resultando mayor a menor EG y menor peso de nacimiento (PN). También es la principal causa de muerte por enfermedad gastrointestinal en neonatos prematuros de muy bajo PN $(<1.500 \mathrm{~g})^{19-22}$. Aunque la mayoría de los casos de ECN son tratados médicamente, se estima que entre 20 y $60 \%$ de los niños pueden requerir cirugía $a^{2,23}$.

La causa de ECN es multifactorial; la prematuridad y baja EG (de la mano con la inmadurez gastrointestinal), alimentación enteral con fórmula láctea, colonización bacteriana, e inclusive la predisposición genética, están frecuentemente implicadas en la patogénesis de esta enfermedad ${ }^{24}$. Todos estos factores pueden contribuir a que el epitelio intestinal prematuro pueda desarrollar una respuesta inflamatoria exagerada, en especial frente a la presencia de bacterias, conduciendo a la destrucción de la mucosa y a una perfusión mesentérica alterada ${ }^{4,24}$. No se ha identificado un microorganismo en particular como agente causal y una amplia gama de patógenos se asocian con ECN, incluyendo bacterias, hongos y virus ${ }^{17}$.

Intensos esfuerzos de investigación en el último decenio han comenzado a dilucidar los fundamentos moleculares de ECN y han identificado varias estrategias biológicas prometedoras que podrían prevenir y/o tratar esta enfermedad en los RNPT ${ }^{4}$. Algunos de estos isolation. The highest risk of death was in umbilical arterial catheter users. Discussion: This ie the first multicenter study that collects local data information. The incidence was similar to that found in medical reports but with a lower mortality. There is no consensus of antibiotic treatment to use. With these results we hope to advance in improving the diagnosis and unify antimicrobial treatments, to reduce morbidity and mortality figures.

Keywords: necrotizing enterocolitis; intestinal microbiota; breastfeeding; microbiology isolation; empirical antibiotic therapy.

tienen que ver con avances en el conocimiento de la inmunología de la mucosa, el rol de los toll-like receptor tipo 4 y la microbiota intestinal ${ }^{9}$.

Muchos estudios han explorado el impacto de la exposición precoz a antimicrobianos y el posterior riesgo de adquirir $\mathrm{ECN}^{25}$, habiéndose establecido que la duración de la exposición a antimicrobianos se asocia con un mayor riesgo de desarrollar esta condición y esta probabilidad se incrementa en $20 \%$ por día de exposición adicional a la antibioterapia empírica. Este riesgo se acrecienta si el tratamiento empírico se prolonga por cinco días o más, llegando a ser tres veces mayor el riesgo con tratamiento antimicrobiano superior a 10 días $^{9}$.

Por otra parte, los niños que necesitan cirugía pueden desarrollar complicaciones post-operatorias, incluyendo dehiscencia de la herida, abscesos intra-abdominales, obstrucción intestinal, estenosis intestinal $(30 \%)$ e intestino corto $(9 \%)^{26,27}$. Además, esta condición aumenta el riesgo, hasta $25 \%$, de morbilidad neurológica con retraso grave del desarrollo neurológico y deterioro psicomotor ${ }^{28,29}$. Los estudios de seguimiento a 18-22 meses plazo mencionan situaciones tan graves como microcefalia y parálisis cerebral ${ }^{18-20}$. Lo señalado previamente lleva a estadías prolongadas en UCIN, altos costos y disminución de la calidad de vida en los sobrevivientes ${ }^{21,30}$. La morbilidad debida a la ECN sigue siendo elevada, con cifras que han permanecido sin cambios durante décadas, a pesar del uso de potentes agentes antimicrobianos $^{30}$ y pese a los avances en técnicas modernas en diagnóstico y tratamientos sofisticados, la mortalidad asociada, paradójicamente, sigue aumentando ${ }^{31}$.

No existen en la literatura médica nacional datos publicados en los últimos 10 años que den cuenta de la magnitud del problema en forma global ni de las características clínicas, microbiológicas y terapéuticas locales para la ECN, por lo que como Comité Consultivo de Infecciones Neonatales de la Sociedad Chilena de Infectología consideramos importante recabar datos nacionales

El objetivo de este estudio fue describir el comportamiento clínico y epidemiológico de los RN de las UCIN de hospitales públicos de Santiago de Chile, que presentaran ECN durante su hospitalización.

\section{Pacientes y Métodos}

Estudio multicéntrico, descriptivo, con reclutamiento prospectivo de todos los pacientes con diagnóstico de ECN definida en base a la 
clasificación de BELL modificada, aplicada en todos los centros participantes. Incluímos todos los pacientes con definición de ECN de todas las EG, de todos los PN y en cualquier estadío de la clasificación de BELL modificada (IA a IIIB). La recopilación de datos se generó desde la historia clínica de los recién nacidos con diagnóstico de ECN en las UCIN de los siguientes hospitales: Complejo Asistencial Dr. Sótero de Río, Hospital San José, Hospital Clínico San Borja Arriarán, Hospital Clínico Dra. Eloísa Díaz I. La Florida, Complejo Asistencial Barros Luco Trudeau, Hospital San Juan de Dios y Hospital Clínico Félix Bulnes, entre los meses de enero y diciembre de 2016, con seguimiento posterior de cada paciente hasta su alta o fallecimiento.

Se realizó la recolección de datos correspondientes a variables epidemiológicas, clínicas, de laboratorio, quirúrgicas y secuelas (detectadas hasta el alta clínica o fallecimiento del paciente). Para efectos de resguardo de la privacidad de los datos, a cada hospital se le asignó un número, presentándose los resultados con esta denominación.

Para efectos de análisis y comparación con datos internacionales se establecieron subgrupos de PN y EG en los RNPT de bajo peso, en los siguientes rangos: $\mathrm{PN}: \leq 1.500 \mathrm{~g}, \leq 1.000 \mathrm{~g}, \leq 750 \mathrm{~g}$ y EG: $\leq 32$ semanas, $\leq 28$ semanas, $\leq 25$ semanas.

En una primera instancia se realizó estadística descriptiva, usando frecuencia y porcentaje, promedios y desviación estándar y medianas con rango intercuartil. El estudio de factores asociados a los desenlaces "cirugía" y "fallecimiento" se realizó en base a análisis univariable y multivariable por regresión logística (para cada desenlace), teniendo como criterio de entrada al modelo de regresión toda asociación univariable con valor $\mathrm{p} \leq 0,10$. El análisis fue realizado utilizando el software SPSS v22.

Este estudio contó con la aprobación de los Comités de Ética Científico de todos los establecimientos participantes e incluyó toma de consentimiento informado a padres o tutores del menor.

\section{Resultados}

Se recolectaron 75 casos de ECN en el período estudiado. Cuarenta pacientes $(53,3 \%)$ pertenecieron al género femenino, un tercio fue pequeño para la edad gestacional (PEG), la mediana de días al diagnóstico fue de 11 días, el promedio de EG fue de $29( \pm 3,8)$ semanas y de PN fue de $1.285 \mathrm{~g}$ ( \pm 661$)$. Como antecedentes perinatales importantes desctacan que casi la mitad de los pacientes sufrió algún grado de hipoxia en el periparto determinado por APGAR menos de 7 al minuto y/o gases alterados con $\mathrm{PH}$ menos de 7 (gases de cordón o a la hora de vida) y un cuarto de los pacientes tenía el antecedente de retardo en
Tabla 1. Datos demográficos generales y antecedentes perinatales de pacientes enrolados con ECN en hospitales públicos de la Región Metropolitana, Santiago, Chile, 2016 (N: 75)

\begin{tabular}{lc}
\hline Antecedentes generales & \\
Género femenino $(\%)$ & $40(53,3)$ \\
\hline PEG (\%) & $23(30,7)$ \\
\hline Promedio EG \pm desv & $29,2 \pm 3,8$ semanas \\
\hline Promedio PN \pm desv & $1.285,6 \mathrm{~g} \pm 661,4$ \\
\hline Mediana de días de vida al dg. (rango IQ 25-75) & 11 días de vida $(8-18)$ \\
Antecedentes perinatales $(\%)$ & \\
RCIU & $19(25,3)$ \\
SHE & $15(20)$ \\
Doppler alterado & $11(14,6)$ \\
RPO & $8(10,6)$ \\
Cerclaje & $8(10,6)$ \\
APGAR $<7$ al minuto & $32(48,5)$ \\
APGAR $<7$ a los 5 min & $14(21,2)$ \\
Gases cordón o primera hora alterados & $28(46,7)$
\end{tabular}

ECN: enterocolitis necrosante. RCIU: retardo del crecimiento intrauterino. SHE: síndrome hipertensivo del embarazo RPO: rotura prematura ovular.

Tabla 2. Distribución de la incidencia de ECN por 1.000 RNV ( $n: 75)$ y letalidad a causa de ECN, enrolados según hospital. Región Metropolitana, Santiago, Chile, 2016

\begin{tabular}{ccc}
\hline Hospitales & $\begin{array}{c}\text { Tasa de incidencia por } \\
\mathbf{1 . 0 0 0 ~ R N V}\end{array}$ & Letalidad (\%) \\
1 & 1,98 & 40 \\
2 & 4,23 & 16,6 \\
3 & 1,82 & 28,5 \\
4 & 1,37 & 0 \\
5 & 2,01 & 16,6 \\
6 & 4,14 & 9,5 \\
7 & 1,49 & 0 \\
\hline ECN: enterocolitis necrosante. RNV: recién nacido vivo. & \\
\hline
\end{tabular}

el crecimiento intra-uterino (RCIU). El detalle completo de los antecedentes perinatales y los datos demográficos generales se describen en la Tabla 1.

La incidencia global fue de 2,6 por $1.000 \mathrm{RNV}$. Las mayores incidencias correspondieron a los hospitales 2 y 6 con 4,23 y 4,14/1.000 RNV, respectivamente. Los pacientes fallecidos a causa de la ECN fueron 14 con una letalidad de $18,6 \%$. Los hospitales 1 y 3 tuvieron la mayor letalidad ( 40 y $28,5 \%$, respectivamente). La distribución de las incidencias de ECN y de las letalidades por cada hospital se presentan en la Tabla 2. 
Tabla 3. Letalidad según peso de nacimiento en pacientes con ECN.

Región Metropolitana, Santiago, Chile, 2016 (N: 14)

\begin{tabular}{lccc}
\hline \multicolumn{4}{c}{ Distribución de fallecidos según peso de nacimiento } \\
Peso nacimiento & $\mathbf{n}$ total & $\mathbf{n}$ fallecidos & Letalidad (\%) \\
\hline $1.500-1.001 \mathrm{~g}$ & 23 & 3 & 13 \\
$1.000-751 \mathrm{~g}$ & 23 & 6 & 26 \\
$\leq 750 \mathrm{~g}$ & 12 & 5 & 41,6 \\
\hline
\end{tabular}

ECN: enterocolitis necrosante.

Tabla 4. Letalidad según edad gestacional, en pacientes con ECN. Región Metropolitana, Santiago, Chile, 2016 (N: 13)

\begin{tabular}{|c|c|c|c|}
\hline \multicolumn{4}{|c|}{ Distribución de fallecidos por edad gestacional } \\
\hline Peso nacimiento & $\mathrm{n}$ total & n fallecidos & Letalidad (\%) \\
\hline 32-29 semanas & 23 & 1 & 4,3 \\
\hline $28-26$ semanas & 25 & 6 & 24 \\
\hline$\leq 25$ semanas & 12 & 6 & 50 \\
\hline
\end{tabular}

ECN: enterocolitis necrosante.

Tabla 5. Antecedentes clínicos de pacientes enrolados con ECN en hospitales públicos de la Región Metropolitana, Santiago 2016 (N: 75)

Antecedentes clínicos

Modo de alimentación al diagnóstico (\%)

$\begin{array}{lr}\text { LM exclusiva } & 17(22,7) \\ \text { Fórmula exclusiva } & 8(10,7) \\ \text { Alimentación mixta } & 40(53,3) \\ \text { LM + LMD } & 5(6,6) \\ \text { LMD exclusiva } & 2(2,7) \\ \text { Sin alimentación } & 2(2,7)\end{array}$

DDV de inicio de la alimentación

Mediana (rango IQ 25-75)

2 días (1-3)

DDV con $100 \mathrm{ml} / \mathrm{kg} / \mathrm{día}$ de tolerancia enteral

Mediana (rango IQ 25-75)

10 días (8-14)

Promedio volumen enteral/kg/ día al diagnóstico (DS)

$96 \mathrm{ml}( \pm 3,2)$

Transfusión de eritrocitos GR previo al diagnóstico (\%)

$32(42,6)$

Uso de antimicrobiano previo al diagnóstico (\%)

$50(66,7)$

Uso de indometacina previo al diagnóstico (\%)

$18(24)$

Uso de prostaglandinas (\%)

$7(9,3)$

Uso corticosteroides prenatales (\%)

$52(69,3)$

Promedio días (DS)
C.U.A
2 días $( \pm 3,2$
C.U.V
3 días $( \pm 3,4)$

LM: leche materna. LMD: leche materna donada. DDV: días de vida. CUA: catéter umbilical arterial. CUV: catéter umbilical venoso.
De los pacientes fallecidos, según peso de nacimiento y edad gestacional, los mayores porcentajes correspondieron para aquellos pacientes $\leq 750 \mathrm{~g}$ con $41,6 \% \mathrm{y} \leq 25$ semanas con $50 \%$. Las Tablas 3 y 4 presentan la distribución de fallecidos según rango de PN y EG.

Cursaron con ECN quirúrgica 31 pacientes (41,3\%), de los cuales, en $22(70,9 \%)$ debió efectuarse una resección intestinal en distinto grado, $16(51,6 \%)$ tuvieron que ser re-intervenidos y $12(38,7 \%)$ fallecieron.

De los antecedentes clínicos más importantes, 24 pacientes $(32 \%)$ recibieron leche materna exclusiva-LME (LM + leche materna donada-LMD) antes de cursar con ECN, recibiendo en su mayoría 40 pacientes $(53 \%)$ alimentación mixta. Se transfundieron de eritrocitos 32 pacientes $(42,6 \%)$ previo al diagnóstico de ECN, 50 pacientes utilizaron antimicrobianos previos al diagnóstico $(66,7 \%)$ y $9(12 \%)$ tuvieron el antecedente de cardiopatía congénita. El resto de los antecedentes clínicos se describen en la Tabla 5.

Los síntomas más frecuentes fueron: distensión abdominal $64(85,3 \%)$, dolor abdominal y residuos gástricos $59(78,6 \%)$. Como signos de mayor gravedad presentaron shock 35 pacientes $(46,6 \%)$, requirieron reanimación con volumen 33 pacientes (44\%), aminas vasoactivas 28 pacientes $(37,3 \%)$ y $20(26,6 \%)$ tuvieron plaquetas $<150.000 \times \mathrm{mm}^{3}$. Todos los neonatos que estuvieron dentro de la clasificación IIIA-IIIB presentaron signos de shock con requerimientos de volumen y/o aminas vasoactivas. En la Figura 1 se puede apreciar la distribución de las ECN de acuerdo con la clasificación de Bell modificada.

A todos los pacientes se les realizó radiografía abdominal, clasificándose de acuerdo con los siguientes hallazgos: normal tres pacientes (4\%), asa fija/asa dilatada/asa centinela/edema de pared intestinal 21 pacientes $(28 \%)$, abdomen blanco dos pacientes $(2,7 \%)$, neumatosis 32 pacientes $(42,7 \%)$, gas en la vena porta un paciente $(1,3 \%)$ y neumoperitoneo 16 pacientes $(21,3 \%)$.

Los antimicrobianos más frecuentemente utilizados, previos al diagnóstico de ECN, fueron ampicilina y amikacina, ocurriendo en 50 pacientes $(66,7 \%)$. De este grupo, 24 pacientes $(48 \%)$ utilizaron $\geq 5$ días de antimicrobianos empíricos y de estos 11 (45,8\%), pacientes fallecieron por ECN.

Se logró aislamiento microbiológico en 34 pacientes $(45,3 \%)$ y en siete de ellos fue el mismo microorganismo en dos fluidos diferentes (orina-sangre, líquido peritonealsangre). Los lugares de aislamiento microbiológico fueron: sangre $21(61,7 \%)$, orina $13(38,2 \%)$, líquido peritoneal cinco $(14,7 \%)$, secreción endotraqueal uno $(2,9 \%)$ y otro lugar dos $(5,8 \%)$. No hubo aislamiento en deposiciones. En 53\% de los casos el microorganismo aislado fue cocáceas grampositiva y de éstos Staphylococcus epidermidis ocupó el primer lugar: 11 pacientes $(32,5 \%)$. 
El segundo microorganismo con mayor frecuencia aislado fue Klebsiella spp. en siete pacientes (20,5\%). En cuatro pacientes $(11,7 \%)$ el aislamiento correspondió a Candida albicans, siendo todos pacientes $\leq 26$ semanas, y la mayoría correspondió al hospital 2. De los pacientes fallecidos en los que se aisló microorganismo (n: 10), en cinco $(50 \%)$ se aislaron bacterias gramnegativas, tres (30\%) fueron especies grampositivas (sólo uno de ellos Staphylococcus epidermidis) y en los otros dos (20\%) se aisló Candida sp. (Figura 2).

Con respecto al tratamiento antimicrobiano para las ECN, se registraron 19 diferentes tipos de esquemas empíricos utilizados al momento del diagnóstico, ya fuese como antibioterapia bi o triasociada. Las combinaciones más utilizadas fueron: cloxacilina + amikacina + metronidazol en 20 pacientes $(30,8 \%)$; vancomicina+amikacina+metronidazol en 18 pacientes $(25,6 \%)$ y cloxacilina + amikacina en 11 pacientes $(10,3 \%)$. En 33 pacientes (44\%) se utilizó un segundo esquema antimicrobiano. En total 33 pacientes $(56 \%)$ utilizaron cloxacilina y $29(38,6 \%)$ utilizaron vancomicina, en distintas combinaciones.

Las principales complicaciones vigiladas hasta el alta hospitalaria y/o fallecimiento fueron malabsorción intestinal e intestino corto, tres casos cada uno (4\%), y obstrucción intestinal, cinco casos $(6,6 \%)$.

Del total de pacientes incluidos en el estudio, 48 (64\%) presentaron la combinación de $\mathrm{PN} \leq 1.500 \mathrm{~g}, \mathrm{EG} \leq 32$ semanas y clasificación de Bell modificada diferente de IA-IB. En base a este subgrupo de pacientes, que son los de mayor riesgo, se realizó un primer análisis multivariable por regresión logística que demostró una tendencia significativa a mayor riesgo de cirugía en pacientes usuarios de catéter umbilical arterial (OR ajustado 8,82; IC95\% 1,43-54,58), PCR > $10 \mathrm{mg} / \mathrm{L}$ (OR ajustado 5,59; IC95\% 1,13-27,55) y aislamiento microbiológico (OR ajustado 8,05; IC95\% 1,29-50,27). Por su parte, un segundo análisis por regresión logística realizado en los mismos pacientes demostró una tendencia significativa a un mayor riesgo de fallecer en usuarios de catéter umbilical arterial (OR ajustado 9,62; IC95\% 1,50-61,74).

\section{Discusión}

El presente es el primer estudio multicéntrico que recopila información de la ECN en nuestro país. Se obtuvo una incidencia de 2,6 por $1.000 \mathrm{RNV}$, similar a lo reportado por la literatura científica; si analizamos cada centro por separado, podemos apreciar que dos centros sobrepasan esta cifra.

La letalidad fue de 18,4\% y aumentó considerablemente si la ECN fue quirúrgica, llegando en estos casos a $38,7 \%$. La letalidad por centro es bastante dispar y

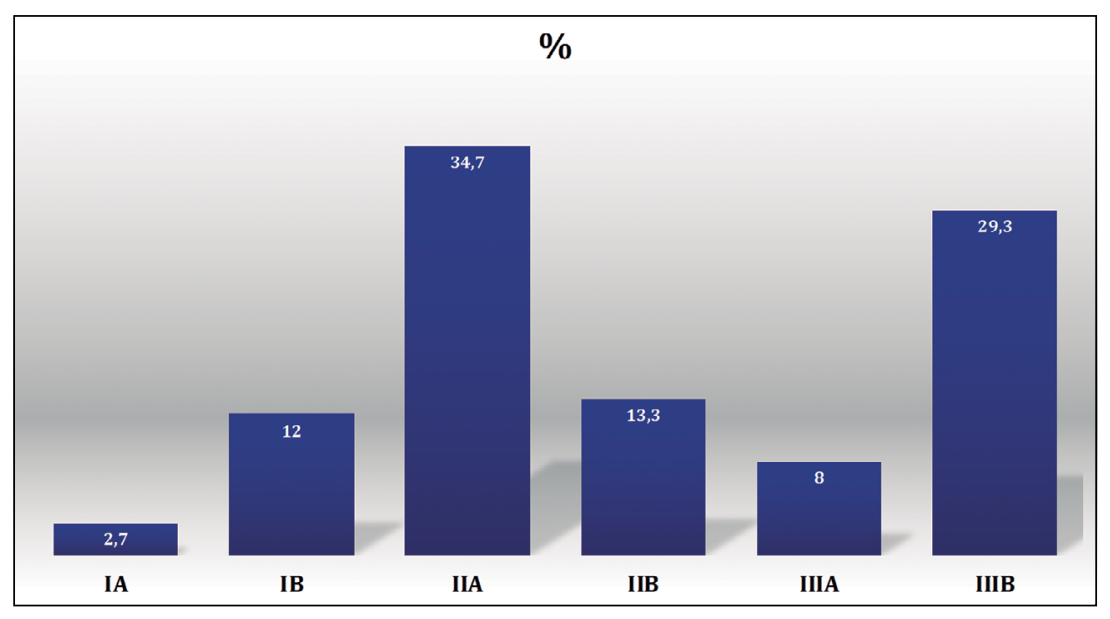

Figura 1. Distribución de los pacientes enrolados con ECN en hospitales públicos de la Región Metropolitana, Santiago, Chile, 2016, de acuerdo con la clasificación de BELL modificada $(n=75)$.

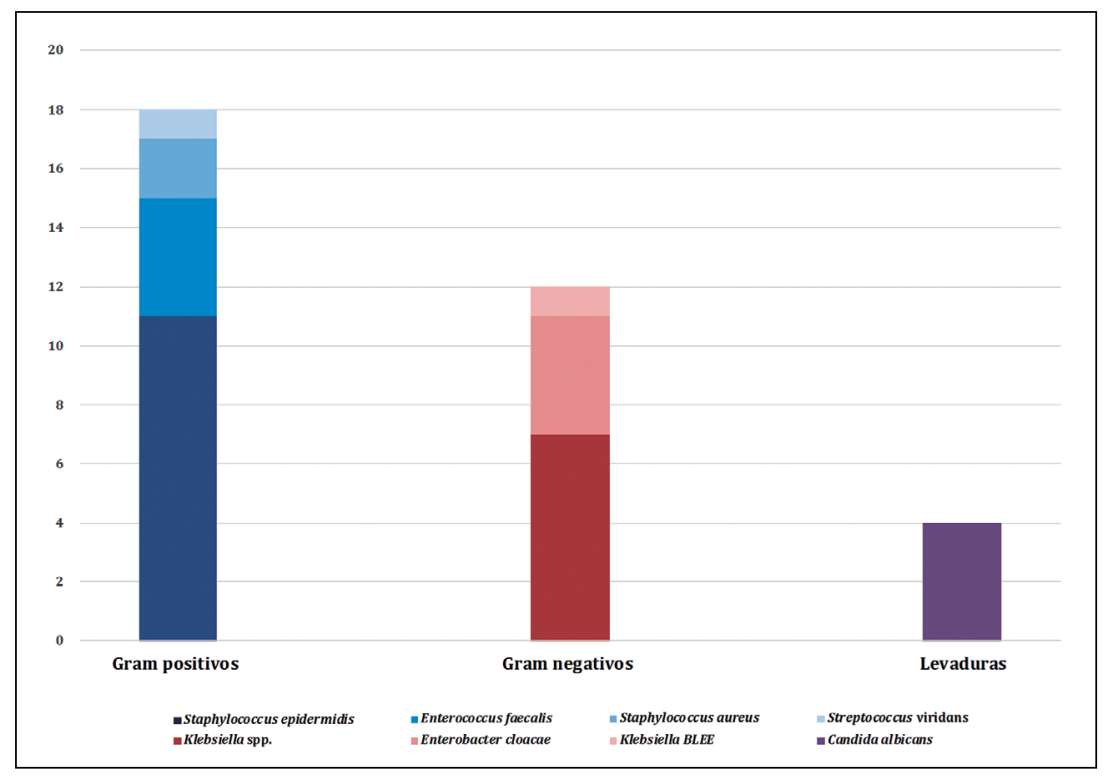

Figura 2. Principales aislamientos microbiológicos de pacientes enrolados con ECN en hospitales públicos de la Región Metropolitana, Santiago, Chile, 2016 (N: 34).

mucho más elevada en los hospitales 1 y 3 . En nuestra serie falleció sobre $40 \%$ de los neonatos $\leq 750 \mathrm{~g} \mathrm{y} \leq 25$ semanas habiendo una clara relación de mayor letalidad a menor EG y menor PN, concordante con la literatura científica $^{28}$.

A pesar de largos años de experiencia en el tratamiento de los pacientes que presentan ECN, la incidencia, letalidad global y el tratamiento se han mantenido prácticamente sin cambios hace varias décadas. La letalidad global y de la ECN quirúrgica en nuestra serie fue algo menor a lo reportado internacionalmente, pero se mantuvo la regla de mayor mortalidad a menor PN y menor EG. 
En nuestro estudio, la mediana de días del diagnóstico está dentro de las dos semanas de vida, cifra que coincide con lo reportado. Llama la atención que $20 \%$ de los pacientes con EG compatible para recibir corticosteroides antenatales, no los recibió, lo cual pudo tener varias causas que no fueron analizadas en detalle. Un reciente metaanálisis demostró que el uso de corticosteroides prenatales a $E G \leq 34$ semanas logró una reducción significativa de la incidencia de $\mathrm{ECN}^{32}$.

Nuestras cifras delatan aún bajos índices de alimentación con LME, pese a que, la LME se ha asociado con 10 veces menor riesgo de ECN en aquellos lactantes que recibieron más de $50 \%$ de su alimentación con $\mathrm{LM}^{33}$. El riesgo de ECN aumenta hasta $21 \%$ por cada $10 \%$ de aumento de alimentación con fórmula en la dieta ${ }^{33}$. La leche materna tiene una gran cantidad de propiedades como son las sustancias bactericidas, inmunológicas, anti-oxidantes y anti-inflamatorias. Además, contiene bacterias comensales que pueden reducir el riesgo de ECN promoviendo una colonización intestinal menos patológica, así como disminución de la respuesta inflamatoria intestinal frente a bacterias patógenas y toxinas ${ }^{34}$.

Al respecto, el uso profiláctico de probióticos entéricos se muestra como una promesa significativa en la prevención de ECN. Una revisión Cochrane concluyó que los probióticos, en el contexto de la prematuridad, previenen la ECN grave y disminuyen la mortalidad por todas las causas. Otra revisión sistemática con meta-análisis más reciente y utilizando estudios adicionales apoyaron estos hallazgos y, a pesar de que existen diferentes regímenes de dosificación en los distintos estudios, se plantea que sería la medida más costo-efectiva y de mayor impacto en estos pacientes ${ }^{9}$. En nuestro estudio, sólo un centro (hospital 5) utiliza probióticos como medida profiláctica en pacientes $<1.500$ g. y/o $<32$ semanas, desde que la vía oral está disponible hasta las 34 semanas. Aún no es posible obtener conclusiones acerca del impacto del uso de probióticos en este centro asistencial debido al bajo número de pacientes registrados.

En cuanto a la relación de transfusiones de eritrocitos y el riesgo de ECN existen controversias ya que, publicaciones iniciales, de metodología retrospectiva, enunciaron que este factor influye en el desarrollo de ECN, sobre todo si el paciente recibe la transfusión mientras se alimenta. Un estudio más reciente determinó que la anemia grave sería responsable de los cambios post-natales en la resistencia vascular esplácnica, en la lesión hipoxémicoisquémico de la mucosa del intestino y posiblemente en la $\mathrm{ECN}$, más que las propias transfusiones ${ }^{35,36}$. A pesar de que casi la mitad de los pacientes presentaba una transfusión previa a la ECN, no se dispuso del antecedente de si se estaban alimentando en forma concomitante.

Destaca como una asociación importante que $66,7 \%$ de los pacientes en esta serie utilizó antimicrobianos previos al diagnóstico de ECN y de éstos $48 \%$ los utilizó durante cinco o más días; casi la mitad de ellos falleció a raíz de la ECN. No se dispone de los datos sobre si este uso fue justificado de acuerdo con factores de riesgo, cultivos positivos, reactantes de fase aguda elevados o sólo fueron indicados en forma empírica. El dato de antibioterapia previa al diagnóstico es importante ya que el desarrollo de la barrera intestinal se altera de forma prolongada a consecuencia de su administración y muchas veces es difícil de recuperar, sobre todo cuando su utilización ocurre en etapas precoces de la vida ${ }^{34,37}$. Los antimicrobianos conducen a la pérdida de la microbiota comensal, con lo cual el microclima intestinal es menos ácido y favorece el crecimiento de las enterobacterias, que en caso de RNPT son especies nosocomiales con patrones de resistencias deletéreos ${ }^{3,4}$.

Habitualmente, la bacteriemia se puede documentar en 20 a $30 \%$ de los niños con $\mathrm{ECN}^{3,6,24}$, cifra más baja que la obtenida en nuestro estudio, la cual incluso pudo estar subestimada, ya que, no tenemos la certeza de que se haya realizado cultivos a todos los pacientes. Existen estudios iniciales que señalan la importancia de la toma del urocultivo, debido a la asociación entre ECN y uropatógenos, no quedando claro muchas veces cuál fue la patología inicial $^{11}$. Lo expuesto permite ver la relevancia de insistir en que la ECN es una enfermedad multisistémica y que, por ello, el aislamiento microbiológico en cualquier zona estéril puede tener importancia para determinar la causa infecciosa de esta afección, y así dirigir en mejor forma la antibioterapia, minimizando sus efectos adversos.

Así como los datos reportados desde los diferentes centros revelaron un alto grado de variabilidad en la terapia antimicrobiana, actualmente no hay consenso ni evidencia en la literatura médica sobre qué régimen de antimicrobianos se debe prescribir para los $\mathrm{RN}$ con $\mathrm{ECN}^{8}$. El tipo de esquema utilizado varía entre UCIN, con poca evidencia para apoyar un régimen sobre otro, ya que hay una falta de datos para hacer recomendaciones formales ${ }^{7}$. Los patrones locales de resistencia microbiana deben guiar la elección de los antimicrobianos ${ }^{3}$. Una revisión Cochrane del año 2014 encontró sólo dos ensayos pequeños, que datan de la década de 1980 y que compararon diferentes regímenes, incluyendo ampicilina, gentamicina y clindamicina ${ }^{38}$. Se encontró una asociación entre clindamicina y un mayor riesgo de estenosis intestinal post ECN, por lo cual hoy en día no se recomienda emplear clindamicina como parte del esquema anti-anaerobio. Recientemente, un estudio de cohorte encontró que la adición de metronidazol a antibacterianos de amplio espectro no previene el deterioro de la ECN establecida y tampoco tuvo impacto en la mortalidad ${ }^{38}$. Sobre la base de estudios más antiguos, la Organización Mundial de la Salud (OMS) recomendó el uso de ampicilina, gentamicina y metronidazol durante 10 días cuando se diagnostica la ECN médica. Sin embargo, 
la ultima Revisión Cochrane disponible, junto con una Revisión Sistemática del Comité de Ensayos Clínicos y Resultados de la Academia Americana de Pediatría han llegado a la conclusión de que no existen pruebas suficientes para efectuar recomendaciones específicas sobre el tipo y la duración de los antimcrobianos empleados en pacientes con $\mathrm{ECN}^{7}$. Todo esto lleva a que varios centros reporten una variedad de hasta 22 régimenes antimicrobianos empíricos para el tratamiento de la $\mathrm{ECN}^{7}$. Una comunicación oral presentada en el IDWeek 2016, New Orleans, E.U.A., mostró la utilización de 12 diferentes tipos de esquemas antimicrobianos empíricos en un solo centro $^{39}$. Nuestro estudio no está alejado de esta situación ya que encontramos también una gran variabilidad de esquemas antimicrobianos empíricos utilizados en los siete hospitales analizados.
El análisis de OR realizado fue pensando en evaluar una tendencia de los factores de riesgo involucrados, pero no son un real reflejo de lo que realmente esté involucrado; para ello planteamos que haría falta continuar realizando investigaciones con el fin de recopilar información en un estudio prospectivo de casos y controles con el fin de obtener factores de riesgo reales.

En síntesis, el presente estudio describe pacientes con ECN en siete UCIN de la Región Metropolitana, Santiago, Chile, y pese a ser una aproximación general, nos entrega información valiosa acerca del comportamiento de nuestras ECN. Es nuestro propósito lograr consensos y directrices para mejorar la sospecha diagnóstica, perfeccionar la prevención y unificar tratamientos antimicrobianos, y así avanzar en reducir las cifras de morbimortalidad de esta patología tan catastrófica.

\section{Referencias bibliográficas}

1.- Thompson A M, Bizzarro M J. Necrotizing enterocolitis in newborns: pathogenesis, prevention and management. Drugs. 2008;68 (9): 1227-38. doi: 10.2165/00003495200868090-00004.

2.- Lin P W, Stoll B J. Necrotising enterocolitis. Lancet. 2006; 368(9543): 1271-83. doi: 10.1016/S0140-6736(06)69525-1.

3.- Gupta A, Paria A. Etiology and medical management of NEC. Early Hum Dev. 2016; 97: 17-23. doi: 10.1016/j. earlhumdev.2016.03.008.

4.- Niño D F, Sodhi CP, Hackam D J. Necrotizing enterocolitis: new insights into pathogenesis and mechanisms. Nat Rev Gastroenterol Hepatol. 2016; 13 (10): 590-600. doi: 10.1038/ nrgastro.2016.119.

5.- $\quad$ Yang J, Wang Z, Feng J, Ai Q, Li L, He $\mathrm{Y}$, et al. Application of laser capture microdissection an 16S rRNA gene plymerasa chain reaction in the analysis of bacteria colonizing the intestinal tissue of neonates with necrotizing enterocolitis. Pediatr Infect Dis J. 2015; 34 (10): e279-89. doi: 10.1097/ INF.0000000000000837.

6.- Möller M J, Paul T, Seeliger S. Necrotizing enterocolitis in premature infants and newborns. J Neonatal Perinatal Med. 2016; 9 (3): 233-42. doi: 10.3233/NPM-16915130.

7.- Blackwood B P, Hunter C J, Grabowski J. Variability in antibiotic regimens for surgical necrotizing enterocolitis highlights the need for new guidlines. Surg infect (Larchmt). 2017; 18 (2): 215-20. doi: 10.1089/sur.2016.163. Epub 2017 Jan 3.

8.- Fan X, Zhang L, Tang J, Chen C, Chen J, Qu $\mathrm{Y}$, et al. The initial prophylactic antibiotic usage and subsequent necrotizing enterocolitis in high-risk premature infants: a systematic review and meta-analysis. Pediatr Surg Int. 2018;34(1):35-45. doi: 10.1007/s00383-0174207-z.

9.- Hodzic Z, Bolock A M, Good M. The role of mucosal immunity in the pathogenesis of necrotizing enterocolitis. Front Pediatr 2017; 5:40. doi: 10.3389/fped.2017.00040.

10.- Zvizdic Z, Heljic S, Popovic N, Alajbegovic-Halimic J, Milisic E, Jonuzi A. Contributing factors for development of necrotizing enterocolitis in preterm in the neonatal intensive care unit. Mater Sociomed. 2016; 28 (1): 53-6. doi: 10.5455/msm.2016.28.53-56

11.- Ward D V, Scholz M, Zolfo M, Taft D H, Schibler K R, Tett A, et al. Metagenomic sequencing with strain level resolution implicates uropathogenic $E$. coli in necrotizing enterocolitis and mortality in preterm infants. Cell Rep. 2016; 14 (12): 2912-24. doi: 10.1016/j.celrep.2016.03.015.

12.- Bowker R M, Yan X, De Plaen I G. Intestinal microcirculation and necrotizing enterocolitis: The vascular endothelial growth factor system. Semin Fetal Neonatal Med. 2018; 23 (6):411415. doi: 10.1016/j.siny.2018.08.008.

13.- Pammi M, Cope J, Tarr P I, Warner B B, Morrow A L, Mai V, et al. Intestinal dysbiosis in preterm infants preceding necrotizing enterocolitis: a systematic review and meta-analysis. Microbiome. 2017; 5 (1): 31 doi: 10.1186/s40168-017-0248-8.

14.- Garg B D, Sharma D, Bansal A. Biomarkers of necrotizing enterocolitis: a review of literature. J Matern Fetal Neonatal Med. 2017; 1-14. doi: 10.1080/14767058.2017.1361925.

15.- Lingappan K, Arunachalam A, Pammi M. Lactoferrin and the newborn: current perspectives. Expert Rev Anti
Infect Ther. 2013; 11 (7): 695-707. doi: 10.1586/14787210.2013.811927.

16.- Maheshwari A. Immunologic and hematological abnormalities in necrotizing enterocolitis. Clin Perinatol. 2015; 42 (3): 567 85. doi: 10.1016/j.clp.2015.04.014.

17.- Autmizguine J, Hornik C P, Benjamin D K Jr, Laughon M M, Clark R H, Cotten C M, et al; Best Pharmaceuticals for Children ActPediatric Trials Network Administrative Core Committee. Anaerobic antimicrobial therapy after necrotizing enterocolitis in VLBW infants. Pediatrics. 2015; 135 (1): e117-25. doi: 10.1542/peds.2014-2141.

18.- Zhang L P, Lei XP, Luo L J, Dong W $\mathrm{B}$, et al. Risk factors for necrotizing enterocolitis in very preterm infants: a case-control study in southwest China. J Matern Fetal Neonatal Med. 2017; 1-6. doi: 10.1080/14767058.2017.1395011.

19.- Neu J, Walker W A. Necrotizing enterocolitis. N Engl J Med. 2011; 364 (3): 255-64. doi: 10.1056/NEJMra1005408.

20.- Pammi M, Suresh G. Enteral lactoferrin supplementation for prevention of sepsis and necrotizing enterocolitis in preterm infants. Cochrane Database Syst Rev. 2020; 3 (3): CD007137. doi: 10.1002/14651858.CD007137. pub6.

21.- Tickell D, Duke T. Evidence behind the WHO guidelines: hospital care for children: for young infants with suspected necrotizing enterocolitis (NEC), what is the effectiveness of different parenteral antibiotic regimens in preventing progression and sequelae? J Trop Pediatr. 2010; 56 (6): 373-8. doi: 10.1093/ tropej/fmq110.

22.- Manzoni P, Meyer M, Stolfi I, Rinaldi M, Cattani S, Pugni L, et al. Bovine lactoferrin supplementation for prevention of necrotizing 
enterocolitis in very-low-birth-weight neonates: a randomized clinical trial. Early Hum Dev. 2014; 90 Suppl 1: S60-S65. doi: 10.1016/ S0378-3782(14)70020-9.

23.- AlFaleh K, Anabrees J. Probiotics for prevention of necrotizing enterocolitis in preterm infants. Cochrane Database Syst Rev. 2014; (4): CD005496. doi: 10.1002/14651858. CD005496.pub4.

24.- Müller M J, Paul T, Seeliger S. Necrotizing enterocolitis in premature infants and newborns. J Neonatal Perinatal Med. 2016; 9 (3): 233-42. doi: 10.3233/NPM16915130.

25.- Neu J, Pammi M. Necrotizing enterocolitis: the intestinal microbiome, metabolome and inflammatory mediators. Semin Fetal Neonatal Med. 2018; 23 (6): 400-5. doi: 10.1016/j. siny.2018.08.001.

26.- Cacho N T, Parker L A, Neu J. Necrotizing enterocolitis and human milk feeding: a systematic review. Clin Perinatol. 2017; 44 (1): 49-67. doi: 10.1016/j. clp.2016.11.009.

27.- Frost B L, Modi B P, Jaksic T, Caplan M S. New medical and surgical insights into neonatal necrotizing enterocolitis: a review. JAMA Pediatr. 2017; 171 (1): 83-8. doi: 10.1001/ jamapediatrics.2016.2708.

28.- Hong CR, Han SM, Jaksic T. Surgical considerations for neonates with necrotizing enterocolitis. Semin Fetal Neonatal Med. 2018; 23 (6): 420-5. doi: 10.1016/j.siny.2018.08.007.

29.- Hickey M, Georgieff M, Ramel S.

Neurodevelopmental outcomes following necrotizing enterocolitis. Semin Fetal Neonatal Med. 2018; 23 (6): 426-32. doi: 10.1016/j. siny.2018.08.005.

30.- Gephart S M, Spitzer A R, Effken J A, Dodd E, Halpern M, McGrath J M. Discrimination of GutCheck(NEC): clinical risk index for necrotizing enterocolitis. J Perinatol. 2014; 34 (6): 468-75. doi: 10.1038/jp.2014.37.

31.- Ng P C. An update on biomarkers of necrotizing enterocolitis. Semin Fetal Neonatal Med. 2018; 23 (6): 380-386. doi: 10.1016/j. siny.2018.07.006

32.- Onland W, De Jaegere A P, Offringa M, van Kaam A. Systemic corticosteroid regimens for prevention of bronchopulmonary dysplasia in preterm infants. Cochrane Database Syst Rev. 2017;1: CD010941. doi: 10.1002/14651858. CD010941.pub2.

33.- Cruz D, Bazacliu C. Enteral feeding composition and necrotizing enterocolitis. Semin Fetal Neonatal Med. 2018; 23 (6): 40610. doi: 10.1016/j.siny.2018.08.003.

34.- Torrazza R M, Ukhanova M, Wang X, Sharma R, Hudak M L, Neu J, et al. Intestinal microbial ecology and environmental factors affecting necrotizing enterocolitis. PLoS One. 2013; 8 (12): e83304. doi: 10.1371/journal. pone. 0083304

35.- Patel R M, Knezevic A, Shenvi N, Hinkes M, Keene S, Roback J D, et al. Association of red blood cell transfusion, anemia, and necrotizing enterocolitis in very low-birth-weight infants. JAMA. 2016; 315 (9): 889-97. doi: 10.1001/ jama.2016.1204.

36.- Neu J, Modi N, Caplan M. Necrotizing enterocolitis comes in different forms: historical perspectives and defining the disease. Semin Fetal Neonatal Med. 2018; 23 (6): 370-3. doi: 10.1016/j.siny.2018.07.004.

37.- Huang X Z, Zhu L B, Li Z R, Lin J. Bacterial colonization and intestinal mucosal barrier development. World J Clin Pediatr. 2013; 2 (4): 46-53. doi: 10.5409/wjcp.v2.i4.46.

38.- Eaton S, Rees C M, Hall N J. Current research in necrotizing enterocolitis. Early Hum Dev. 2016; 97: 33-9. doi: 10.1016/j. earlhumdev.2016.01.013.

39.- Corey Lance, Shasha Bai, Holly Maples, Mariam Khan, Nada Harik, Jingyun Li, Dawn Weiss. Antibiotic therapy in patients with necrotizing enterocolitis in the Neonatal Intensive Care Unit: a quality improvement project. Open Forum Infectious Diseases, Volume 3, Issue suppl_1, December 2016. doi. org/10.1093/ofid/ofw172.1482. 